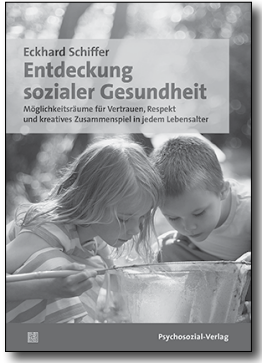

\section{Schiffer Eckhard}

Entdeckung sozialer

Gesundheit

Möglichkeitsräume für

Vertrauen, Respekt und kreatives Zusammenspiel in jedem Lebensalter

Gießen: psychosozial, 2021, $321 \mathrm{~S} ., € 24,90$

Seinen Beitrag zu "Salutogenese kennen und verstehen"(Meier-Magistretti et al. 2019) übertitelte der Autor mit der Frage "Gibt es (k)eine soziale Salutogenese?"

Vielfältige und umfassend begründete Antworten stellt er nun in "Entdeckung sozialer Gesundheit"vor und lädt "Leserinnen und Leser herzlich zu einer spannenden und anregenden Entdeckungsreise ein".

Diese Einladung anzunehmen lohnt sich. Der Autor schöpft aus dem Vollen: Sein reicher Erfahrungsschatz aus 55 Jahren Berufserfahrung als Arzt und Therapeut, als Wissenschaftler, aus Alltagserfahrungen, aus vertieften Einblicken in andere
Berufsfelder und seine schriftstellerische Tätigkeit führen zu sprudelnden, fachlich hochwertigen und gut verständlichen Texten auf der Entdeckungsreise zur sozialen Gesundheit.

Diese Reise ist in fünf größere Etappen aufgeteilt, die ihrerseits verschiedene kleine Etappen umfassen. Das gut gegliederte und ausführliche Inhaltsverzeichnis bietet einen hilfreichen Überblick über die Etappenziele und erleichtert gezielt einzelne davon, entsprechend eigenem Interesse und Vorwissen, auszuwählen. Das Buch ist durchgängig gut gestaltet; zahlreiche Fotos lockern die Texte auf.

Im ersten Teil der Reise stehen beglückende Begegnungen und kreativer, schöpferischer EigenSinn im Zusammenspiel im Vordergrund - Stichworte: Lächelspiele, die Welt der Klänge und Rhythmen, Dialog(e). Dem Salutogenese-Modell, dem Doppelaspekt sozialer Gesundheit und seiner körperlichen Auswirkungen ist die zweite Etappe gewidmet. Die Entwicklung des Kohärenzgefühls wird an der Frage erläutert "Warum Huckleberry Finn ein ausreichendes Kohärenzgefühl entwickeln konnte". Thema des dritten Teils sind die 
biografischen Einflüsse auf die Entwicklung sozialer Gesundheit - aufgezeigt am Beispiel der biografischen Hintergründe von Anders Behring Breivik und Hape Kerkeling und an den Nachwirkungen von staatlicher Kontrolle des dialogischen Eigen-Sinns durch das Nazi-Regime. Die vierte Etappe führt durch die Praxis und die Erfahrungen mit sozial-salutogenen Projekten: Baby-Begegnung in Kita und Schule, sozial-salutogener Schulunterricht, Singen und Musizieren, klassen- und schulenübergreifende Projekte. Im letzten Teil geht es um Orte und Nicht-Orte: Auf-/Wiederfinden sicherer Orte, Spielplätze, die Wahrnehmung von Orten in der Natur mit allen Sinnen.

Die Reise endet nachdenklich mit dem Kapitel "Die Corona-Pandemie als Stresstest für soziale Gesundheit", das Eckhard Schiffer dem Buch aus aktuellem Anlass beigefügt hat.
Auch wer über wenig theoretische Vorkenntnisse zur Salutogenese und zu wissenschaftlichen Erkenntnissen aus Neurobiologie und Säuglingsforschung verfügt, erfährt in diesem Buch, worauf das heutige Verständnis über Einflüsse auf die Entwicklung von sozialer, körperlicher und seelischer Gesundheit beruht. Die vielen praktischen Beispiele illustrieren, was Mütter, Väter, Großeltern, Fachpersonen in Spielgruppe und Kita, in der Schule, in der Arztpraxis, d. h. dort wo sie Kindern - Menschen - in „Möglichkeitsräumen“, im „Zusammenspiel", mit ihrem „Eigen-Sinn" begegnen, zur Entwicklung von (sozialer) Gesundheit beitragen können. Der Autor erachtet es denn auch als hilfreich, sich erst mit den Beispielen und im zweiten Schritt mit der Theorie zu beschäftigen.

Kathrin Keller-Schuhmacher DOI 10.2378/fi2022.art06d 